\title{
Human Genetics and Congenital Pyloric Stenosis
}

Until the last 50 years Congential Pyloric Stenosis had a high mortality rate - and it is only since Rammstedt devised his relatively simple and quick operation that the case mortality has fallen. The condition is still the commonest disorder in Britain in children requiring surgery. This is pointed out by Carter *, who draws attention to the fact that since 1923 sufficient numbers of children with Pyloric Stenosis have now survived to found families from which the genetic factors concerned may be studied. In his article Carter considers evidence from studies of twins and has collected figures from relatives of 174 male patients and 48 females. He concludes that the well-known predominance of males over females is unlikely to be due to sex-linked inheritance. He suggests that the predisposing genotype has probably two components, first a common dominant gene, and second a sexmodified multifactorial background. This paper is one of a number of important contributions on Human Genetics published by the British Medical Council. Though necessarily of general interest these articles have bearings upon gastroenterological and metabolic disorders as for instance in Aminoaciduria. Here its relationship to Wilson's Disease, and the possibility that cirrhosis of the liver in children may be more often due to the biochemical changes of Wilson's Disease than is thought, is intriguing.

Of the many other papers those on Mongolism, Finger-Print Patterns and particulary that on Pharmacogenetics make fascinating reading. In the last of these the value of drugs as a tool for investigating biochemical genetics, especially enzyme systems controlled by allelic genes is exemplified by a review of the individual variations to isoniazid, barbiturates with their action in precipitating porphyria, Hydrogen peroxide, salisyclates and others. The inherited variations of Human Plasma Proteins as detected electrophoretically have vital importance in clinical medicine as shown for example by the growing knowledge of agammaglobulinaemia; this subject is discussed by Harris who refers to some twelve human plasma proteins and their variants. Inherited variations in the leucocytes is another aspect of Cytogenetics which is likely to lead to increasing knowledge perhaps in the direction of elucidating something of the problem of neoplasia.

T.H.

* Carter, C. 0.: Brit. Med. Bull. 1961, Vol. 17, No. 3, p. 251. 